\title{
A Catalogue of Ultraviolet Observations of Chromospherically Active Binary Stars
}

\author{
C. LA DOUS ${ }^{1}$ AND ALVARO GIMÉNEZ ${ }^{2}$
}

\author{
${ }^{1}$ IUE Observatory, ESA, Villafranca del Castillo, Apartado 50727, 28080 Madrid, Spain \\ ${ }^{2}$ LAEFF, INTA, Villafranca del Castillo, Apartado 50727, 28080 Madrid, Spain
}

\begin{abstract}
We present the recently-published IUE-ULDA Access Guide on Chromospherically Active Binary Stars which should be of interest for the interpretation and analysis of EUV data obtained for this type of objects. We provide background information on both high and low resolution $I U E$ spectra of chromospherically active late-type binary stars that have been taken until the end of 1992. Physical information on all systems, arranged by variable star name, together with characteristics of the individual exposures, the position of the observation in the orbital light curves, and an average low resolution ultraviolet spectrum are given.
\end{abstract}

Many of the sources of EUV radiation are cool late-type stars closer than $100 \mathrm{pc}$. Many of them (those having spectral types later than F5) show indicators of solar-like magnetic activity, such as photospheric spots, chromospheric emission, coronal X-ray and radio emission, as well as flare activity (see e.g., Guinan \& Giménez 1993). Apart from very young stellar objects, such as $T$ Tauri stars and related pre-main sequence stars, those with the highest activity levels are close binary systems with cool, G- to M-type, components. Otherwise, the majority of solar-like stars in the Sun's neighborhood are relatively old and have activity levels comparable to that of our Sun.

The currently accepted general picture of the nature of active late-type binaries is based on the emergence of magnetic flux tubes producing active regions in analogy to those in the Sun with spots, active chromospheres and coronae. The combination of deep convective envelopes with magnetic fields and forced stellar rotation lies at the origin of this kind of stellar activity. The strength of the indicators is expected to increase as a function of stellar rotation and of the depth of the convective envelope.

The most prominent groups among the late-type chromospherically active binary stars are those known under the names of RS CVn stars (Hall 1976) - where the stellar components are dwarfs subgiants and giants of spectral type $\mathrm{F}$ to $\mathrm{K}$ (Hall 1981)-and BY Dra stars (Bopp \& Feckel 1977) -where always at least one component is of type dK or dM. Observationally they are characterized by strong strong CaII $\mathrm{H}$ and $\mathrm{K}$ emission. Following the solar analog, these emissions may be identified with enhanced chromospheric emission from plage-like regions and the chromospheric network. Therefore, emission lines which originate in the same stellar region as the Ca II resonance lines but lie at ultraviolet wavelengths (as a large number of them do) should be observable with a better contrast with respect to the continuum flux. The most interesting lines in this context are the chromospheric emission features of $\mathrm{MgII} \mathrm{h}$ and $\mathrm{k}$ at $2800 \AA$ (see Budding and Giménez 1982; Smith et al. 1991) and the transition region line emissions of NV at 1240 $\AA$, SiII at $1400 \AA$, and CIV at $1550 \AA$.

Active late-type binaries are key sources of information on the physical mechanisms responsible for coronal and chromospheric activity. The IUE on Chromospherically Active Binary Stars (ESA SP-1181, 1994) was originally compiled in order to facilitate usage of the information now contained in the IUE Archive; we call attention to it in the context of $E U V E$ astronomy for the interpretation and analysis of $E U V E$ data obtained for these stars. In addition to details on the available observations, it provides background 
information on each system. The object list used is the one provided by Strassmeier et al. (1993). For each object we provide plots of the average low-resolution IUE spectrum and, whenever available, the high-resolution profile of the $\mathbf{M g}$ II line. Various tables provide details on each $I U E$ image (camera, image number, spectral resolution, date and time of observation, FES magnitude, orbital phase, and image quality) and physical parameters (alternative stars names, coordinates, the linear ephemeris, system parameters, activity parameters and photometric parameters from different catalogues.

\section{REFERENCES}

Bopp, B. W. \& FECKEL, F. C. 1977, Binary incidence among the BY Draconis variables, A\&A, 82, 490

BudDing, E. \& GIMÉNEZ, A. 1982, Stellar activity in the short period subgroup of the RS CVn systems, Third European IUE Conference, ed. E. Rolf et al., ESA SP-176, 169

GUINAN, E. F. \& GMÉNEZ, A. 1993, in The Realm of Interacting Binary Stars, ed. J. Sahade et al., Dordrecht: Kluwer, 51

HALL, D. S. 1976, The RS CVn binaries and binaries with similar properties, in Multiple Periodic Phenomena in Variable Stars, IAU Colloq. 29, ed. W. S. Fitch, Dordrecht: Reidel, 287

HALL, D. S. 1981, The RS Canum Venaticorum binaries, in Solar Phenomena in Stars and Stellar Systems, ed. R. M. Bonnet \& A. K. Dupree, Reidel, Dordrecht/Holland, 431

Smith, G. H., Burstein, D., Fanelli, M. N., O'Connell, R. W. \& Wu, C. C 1991, On the utility of low resolution IUE spectroscopy of the $2800 \mathrm{~A}$ MgII lines as a stellar chromosphere indicator, $\mathrm{AJ}, 101,655$

Strassmeier, K. G., Hall, D. S., Feckel, F. C. \& Scheck, M 1993, A catalogue of chromospherically active binary stars second edition, A\&AS, 100, 173 